\title{
Modern Treatment of Community-Acquired Pneumonia: A Scientific Justification or Belief in a Panacea?
}

Igor Klepikov*

Department of Pediatric surgery, USA

*Corresponding author: Igor Klepikov, Department of Pediatric surgery, 2116, NE, 27st, Renton, WA, 98056, USA, Email: igor.klepikov@yahoo.com

\section{Letter to Editor}

Volume 3 Issue 1

Received Date: May 21, 2018

Published Date: May 30, 2018

\section{Introduction}

The results of scientific research are the basis and intellectual potential of practical medicine and its main driving force of improvement and development. When the results of such research become clear and generally accepted, they can usually acquire the features of direct directives for practical application. As a rule but unfortunately not always. Modern ideas about the nature of acute pneumonia (Community-Acquired Pneumonia, CAP) and the principles of its treatment are a striking example of the oblivion the results of classical and very important scientific research. The paradox of this example is that the materials of most of these studies are familiar to medical professionals from the University bench. At the same time, there is no mention or discussion of important fundamental sources in modern literature describing CAP.

In this regard, it is necessary to note some medical and biological axioms and postulates, which significantly change the understanding of the nature and mechanisms of CAP development. These materials relate both to the role of the lungs in the body and to the process of nonspecific inflammation. Such a reminder is especially necessary in the light of the fact that CAP is the only process with localization in the vascular system of a small circle of blood circulation from all nosologies of nonspecific inflammation.

Firstly, one of the most important functions of the lungs is not only ventilation and gas exchange, but also the regulation of blood circulation in the body. A prerequisite for the synchronous operation of the pulmonary cardiac apparatus is the equality of volume blood flow in the right and left heart. The slightest shift of this equilibrium is accompanied by an Autonomous reflex correction. This stabilization is possible due to the ability of the body to constantly maintain the opposite proportions of systemic pressure in small and large circles of blood circulation (reflex of Schwigk).

Secondly, vascular reaction is the basis of anatomical transformation of tissues in the zone of non-specific inflammation of any location. This reaction has been studied in detail and has strictly consistent stages of its development.

Thirdly, non-specific inflammation regardless of the pathogen is accompanied by classical signs, which were described by Celsius and Galen many centuries ago. Features changes in blood flow in the area of inflammation and increased vascular permeability lead to signs such as heat, redness, swelling. Shifts in the chemical composition of tissues give irritation to nerve structures in this zone and are accompanied by pain and reflex reactions. However, the clinical picture to a greater extent is determined by the location of inflammation and the development of the fifth sign, loss of function.

Fourth, the intensity of the inflammatory response is determined on the one hand, the reactivity of the body, and on the other hand, its protective and adaptive capabilities. These two antagonistic actions have exclusively individual character. If there was a possibility of their accurate measurement, the result of such a study would be a scale similar to the comparison of fingerprints.

Fifth, CAP has never been classified as a contagious infectious disease. In modern literature on this topic cannot find statements about the need for compliance with special sanitary and epidemiological measures in patients with CAP. Meanwhile, in recent years, CAP is increasingly considered and even classified as an 


\section{Open Access Journal of Pulmonary \& Respiratory Sciences}

infectious disease. In this connection it is necessary to remind well-known features of etiology of CAP which contradict exclusively infectious nature of this disease.

a) CAP does not have one permanent pathogen.

b) Various representatives of symbiotic micro flora can act as an agent of CAP.

c) Prolonged stay in an organism of healthy people of the most virulent microorganisms, including antibioticresistant strains (latent carrier) testifies that for the beginning of CAP existence of the causative agent is insufficient only. These facts also contradict one of the existing theories of the origin of CAP by simply inhaling microbes into the lungs.

Some of the above scientific facts may be considered "gold Fund" of medical science and a reminder of them is hardly a revelation for professionals. Ideas about nonrespiratory lung functions and features of the inflammatory process are important for practical medicine and require due consideration in the justification of the principles of treatment. . At the same time, despite the lack of convincing arguments about the exclusive dependence of CAP on its pathogen, antibacterial measures remain the leading principle of its treatment. It is no secret that approaches to the treatment of CAP actually repeat medical care for diseases such as angina (tonsillitis), otitis media, furunculus, pyelonephritis and others that are fundamentally different from pneumonia. In addition, initial treatment ("antibiotics alone") usually consists of the distribution of the same antibiotic among this diversity of patients.

Neglect of specific methods of influence on pathogenetic mechanisms of development of CAP significantly reduces the amount of assistance to such patients, leaving emerging shifts only for the potential of protective and adaptive forces of the organism. In this regard, it should be noted that the long-term practice of antibiotics brought out of the usual equilibrium symbiotic micro flora of the body and gave rise to undesirable phenomena. So, periodically there is a change of leadership among agents CAP. The list of antibioticresistant strains is growing steadily. Reducing the effectiveness of drugs in use forcing pharmacists to develop new more effective antibiotics. However, the creation of new drugs is due to changes in conditions and a decrease in the effectiveness of previous antimicrobial forms. This development of new antimicrobials is determined by the appearance of a specific purpose and cannot be carried out in advance.

Maintaining current views on the nature of CAP and its treatment will not change the situation and will not be able to improve the final results. Moreover, a further increase in antibiotic-resistant strains and a gradual reduction in the antimicrobial effect of drugs will only support a negative trend in CAP treatment outcomes.

Meanwhile, the possibility of effective treatment of even the most aggressive forms of CAP not only exists. Such a complex of pathogenetic approaches to the treatment of CAP was scientifically substantiated, confirmed by additional studies and, most importantly, tested in clinical practice [1]. A detailed description of the work done and its results are presented in a separate book [2].

It is important to note that the proposed CAP doctrine and the resulting treatment approaches do not require the development of new drugs or significant additional funding. The most difficult stage in the dissemination and popularization of the new doctrine of the disease is a radical change of views and beliefs. For specialists with experience and persistent professional views, such retraining is a difficult barrier.

It is necessary only in accordance with the legislative requirements of specific government bodies and commissions to conduct the necessary testing and approval of other approaches to the treatment of CAP and change the curriculum of training doctors in this section. The implementation of these plans will spread the gains already made, but the formal application of these results will require the assistance of specialists in administrative and legal work.

In this regard, this manuscript is an appeal to a wide range of professionals who have both direct and indirect relation to the problem of inflammatory lung diseases. The implementation of the proposed project is necessary primarily for future patients with a CAP. That is why I very much count on professional support and real assistance in promoting this prospect.

\section{References}

1. Klepikov I (1989) Acute pneumonia and its purulent and destructive complications in children in the midst of a major industrial centre of Western Siberia. Dissertation for the degree of doctor of medical science Leningrad 989.

2. Klepikov I (2017) Acute pneumonia: a new look at the old problem. Lambert Academic Publishing ISBN (978-3-330-35250-6). 